\title{
A Voice Coil Powered Controllable Micro-Jet Injection System
}

\author{
Kai Chen', Laiwu Miao1, Zhigang Feng2 \\ ${ }^{1}$ School of Mechanical Engineering, Hangzhou Danzi University, Hangzhou, China \\ ${ }^{2}$ Department of Mechanical Engineering, University of Texas at San Antonio, San Antonio, USA \\ Email: kchen@hdu.edu.cn
}

Received 5 August 2015; accepted 11 September 2015; published 14 September 2015

Copyright (C) 2015 by authors and Scientific Research Publishing Inc.

This work is licensed under the Creative Commons Attribution International License (CC BY).

http://creativecommons.org/licenses/by/4.0/

(c) (i) Open Access

\begin{abstract}
A medical device of micro-jet injection for drug delivery is described in this paper. The device is powered by a Lorentz force driver (or voice coil motor, VCM) and is able to perform pulsed injection through controlling the direction of the current passing through the device. The driving force and the resulting injection pressure are also controllable through control of the current intensity of the VCM. A physical model was established by combining the existing jet injection model with the relationship of the driving force obtained from a finite-element-method (FEM) analysis, and was verified by experimental measurements. The numerical calculation of the physical model reveals the relationship between the injection pressure and the current intensity of VCM under system conditions. In normal cases, the injection dose can be varied. Thus the relationship between the current intensity of VCM and the dose value was numerically obtained under the condition for the maximum injection pressure to be above a threshold value. These results can be used for optimization of the device.
\end{abstract}

\section{Keywords}

Jet Injection, Micro-Jet, Trans-Dermal Drug Delivery

\section{Introduction}

Over the years, conventional hypodermic needles have been an effective way of subcutaneous drug delivery. However, the process of needles piecing into the skin produces pain causes panic and extra pressure. A common needle-free jet injector employs a high-velocity liquid stream generated either by a spring or compressed gas to penetrate into the skin [1]. It has a number of advantages such as simpler operation, better absorption, elimination of infection, etc. Most commercial jet injectors complete the injection in one shot, and are not able to 
achieve sustained and controlled release of drug delivery. The driving force that powers the jet injector is often fixed so that the injection depth is uncontrollable. When the injection depth is too deep and the jet touches nerves under the skin, it is often accompanied by a strong sense of pain [1]. In addition to springs and compressed air, alternative driving forces have been explored in order to transform the one-time jet injection into an intermittent micro-jet injection. The micro-jet injection system was first proposed by Arora, et al. [2]. It applied a piezoelectric stack actuator as the driving power. Owing to the small displacement of the piezoelectric actuator output, the single injection dose is very small and the injection depth is effectively controlled [2]-[6]. A pulsed micro-jet can also be produced using high-voltage electrode discharged in the liquid. Bubbles are created to drive the liquid out of the nozzle [7] [8]. A recent study applied a pulsed laser irradiation into the liquid to generate driving bubbles to achieve micro-jet drug delivery [9]. This kind of continuous pulsed micro-jet injection for trans-dermal drug delivery has features such as less invasiveness, sustained drug delivery, controlled drug release and so on and so forth.

Taberner et al. [10] reported a jet injector powered by a voice coil linear actuator which is composed of rare earth permanent magnets as the core and a winding pack of coil as the moving actuator. A high power density capacitor was used to discharge electricity that passes through the coil to produce the electromagnetic force (Lorentz force) high enough to obtain a high velocity jet. Because the driving force (Lorentz force) can be controlled and regulated through the current intensity and the time passing through the coil, the resulting impinging pressure of the jet and also the penetration depth are controllable through the manipulation of these parameters applied to the coil. Recently, Taberner et al. [11] demonstrated a control strategy that can change and optimize the jet speed through feedback control of piston displacement during the course of jet injection. However, the current jet injection systems based on the voice coil actuator so far can only be used for a single-shot injection. A continuous micro-dosing system is thus necessary for the controlled release of drug delivery in clinical applications. This paper presents a continuous micro-jet injection system based on the voice coil actuator. The relationship between the jet impinging pressure and the current intensity applied on the voice coil was established for different injection volumes based on an existing mathematical model as well as results from a FEM analysis of magnetic field under system conditions.

\section{The System Structure and Working Principle}

A needle-free jet injection system includes an ampoule, a driving device and its fixture. The ampoule is mainly used for inhaling, reserving drugs, and accomplishing flow ejections. The driving device provides driving force for the piston in the ampoule to expel the liquid out of a small orifice at the front end of the ampoule. As shown in Figure 1, the current continuous micro-jet injection system includes an injector, an ampoule, a VCM (voice coil motor) drive, a control system, a power supply and some moving and support structures. The VCM consists of a core pack of permanent magnets and a moving coil, both of which are surrounded by a shell. The piston inside the ampoule connects to the plunger of VCM through a coupler. Two precision check valves on the ampoule function as pump valves to suck the liquid from the drug storage and expel the liquid from the front end of the ampoule, and then out of the orifice at the front end of the injector. An inelastic hose connects the front end of the ampoule to the back end of the injector.

In operation, upon accepting the signal from the actuator of the injector, the control system triggers the power supply to apply high voltage on the VCM for a period of preset time. The moving coil in the VCM under Lorentz force pushes the piston inside the ampoule. The check valve on the orifice opens while the other check valve that connects to the drug storage closes. The high-pressure liquid flows through the hose into the injector and forms a high speed jet after coming out of the orifice. After injection, the control system provides a reverse direction current for the VCM. The piston then moves back. The two check valves operate just in the opposition of the injection, and the liquid flows from the drug storage to the ampoule, in preparation for the next injection. Each cycle of an injection completes instantaneously and can be programmatically regulated by the control system.

\section{The Physical Model and Calculation}

Many studies have been performed in order to establish predictive models that relate the penetration shape and depth with the injection power and volume during jet injections [12]-[14]. However, these models are difficult to be directly utilized in an existing device due to variation of skin property and system conditions. A common result 


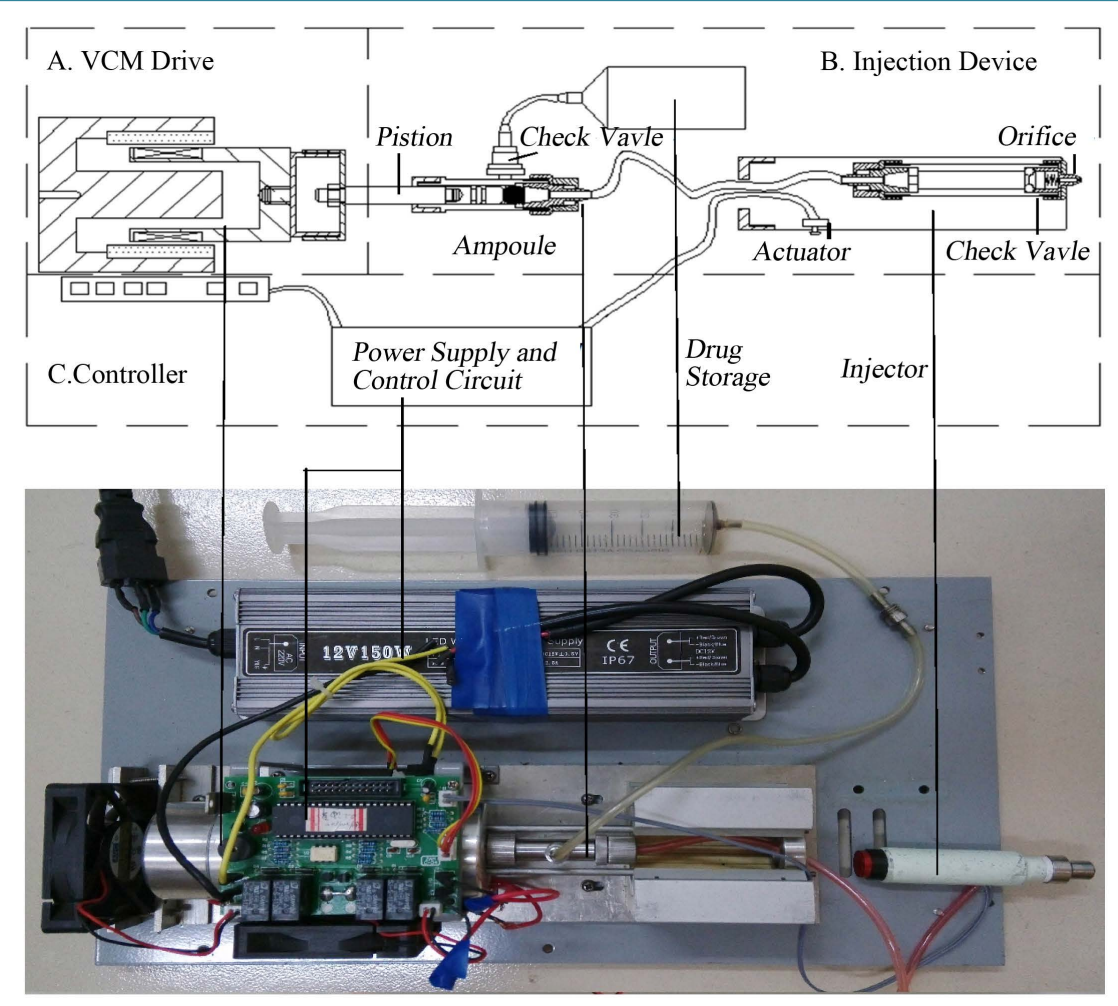

Figure 1. The structure diagram of the continuous micro-jet injection system.

reveals that the injection pressure plays an important role in the performance of the jet injection process. It is reported that the maximum injection pressure in the initial moment needs to reach above a threshold before the jet is able to erode and penetrate into the skin [15]. The authors have developed a quasi-steady physical model to calculate the dynamic injection pressure for a given driving force [16]. By solving the force balance and the mass and energy conservation established separately for driving piston and for injection liquid, Equation (1) was obtained to express the piston movement and the injection pressure as coupled functions of time.

$$
\left\{\begin{array}{l}
\frac{\mathrm{d}^{2} x_{p}}{\mathrm{~d} t^{2}}=\frac{F}{m_{p}}-\frac{A_{p} p}{m_{p}}-\frac{\pi D b \theta\left(p+p_{c}\right)}{m_{p}} \\
\frac{\mathrm{d} p}{\mathrm{~d} t}=\frac{(E+p) \frac{\mathrm{d} x_{p}}{\mathrm{~d} t}-\frac{E A_{0}}{A_{p}} \sqrt{\frac{2 p \frac{\mathrm{d} x_{p}}{\mathrm{~d} t}}{\rho_{0} \frac{\mathrm{d} x_{p}}{\mathrm{~d} t}+\frac{64 \mu L}{D^{2}}}}}{L-x_{p}}
\end{array}\right.
$$

where $x_{p}$ is the total displacement of the piston, $F$ the electromagnetic driving force of the VCM, and $p$ the hydraulic pressure on the piston surface. Other symbols are shown in Figure 2, and are explained in Table 2 of next section. The first equation represents a force balance where the three terms on R.H.S are driving force, liquid resistance, and friction resistance on the piston ring, respectively. The second equation is actually a flow rate variation under the control volume of injection liquid, where the velocity at the orifice is represented by the hydraulic pressure across a streamline under the condition of viscous loss (the part of square root).

The injection pressure of the jet, $p_{s}$, in the surface of skin can be calculated according to Bernoulli principle, where $v$ is the speed of jet out of the ampoule.

$$
p_{s}=\frac{1}{2} \rho v^{2}
$$


In a separate paper, the authors have calculated the electromagnetic force through FEM simulation with the commercial software Maxwell 3D for each separate displacement of the coil movement, and for different values of current intensity that pass through the VCM [17]. Since magnetic intensity $B$ continuously changes in the course of coil movement, 9 discrete points of displacement with $5 \mathrm{~mm}$ of interval along the moving distance of the coil were set up for each round of calculation. Figure 3 shows the simulation image of magnetic field intensity. The resultant electromagnetic forces of coil under different conditions of current intensity were obtained and were shown in Figure 4.

The discrete data of electromagnetic force is further processed through a quadratic curve fitting method to obtain the continuous relationship between electromagnetic force and the displacement under different values of current intensity. The fitting results were also shown in Figure 4. The following relationship was established that approximates the electromagnetic force $F(\mathrm{~N})$ as a function of piston displacement $x(\mathrm{~mm})$ and the current intensity $I(A)$. The Equations (1) to (3) can be combined to derive the injection pressure varies with time under system conditions.

$$
F=-(0.01 I+0.06) x^{2}+4 x+10 I
$$

\section{The Calculation Results, Verification and in Vivo Test}

Equations (1)-(3) can be solved by the classical Runge-Kutta integration method. The main parameters of the VMC used for calculation and also for the device are shown in Table 1. The other system parameters used for calculation are listed in Table 1.

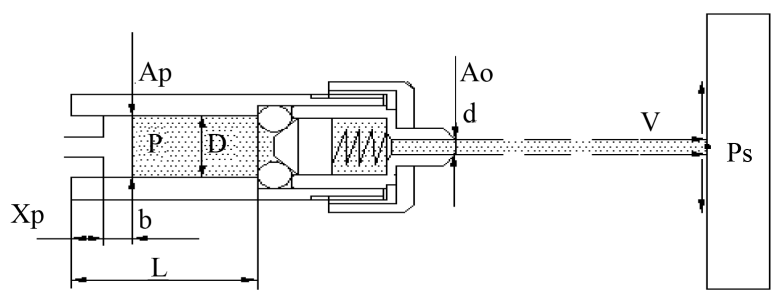

Figure 2. The physical model of the injection flow.

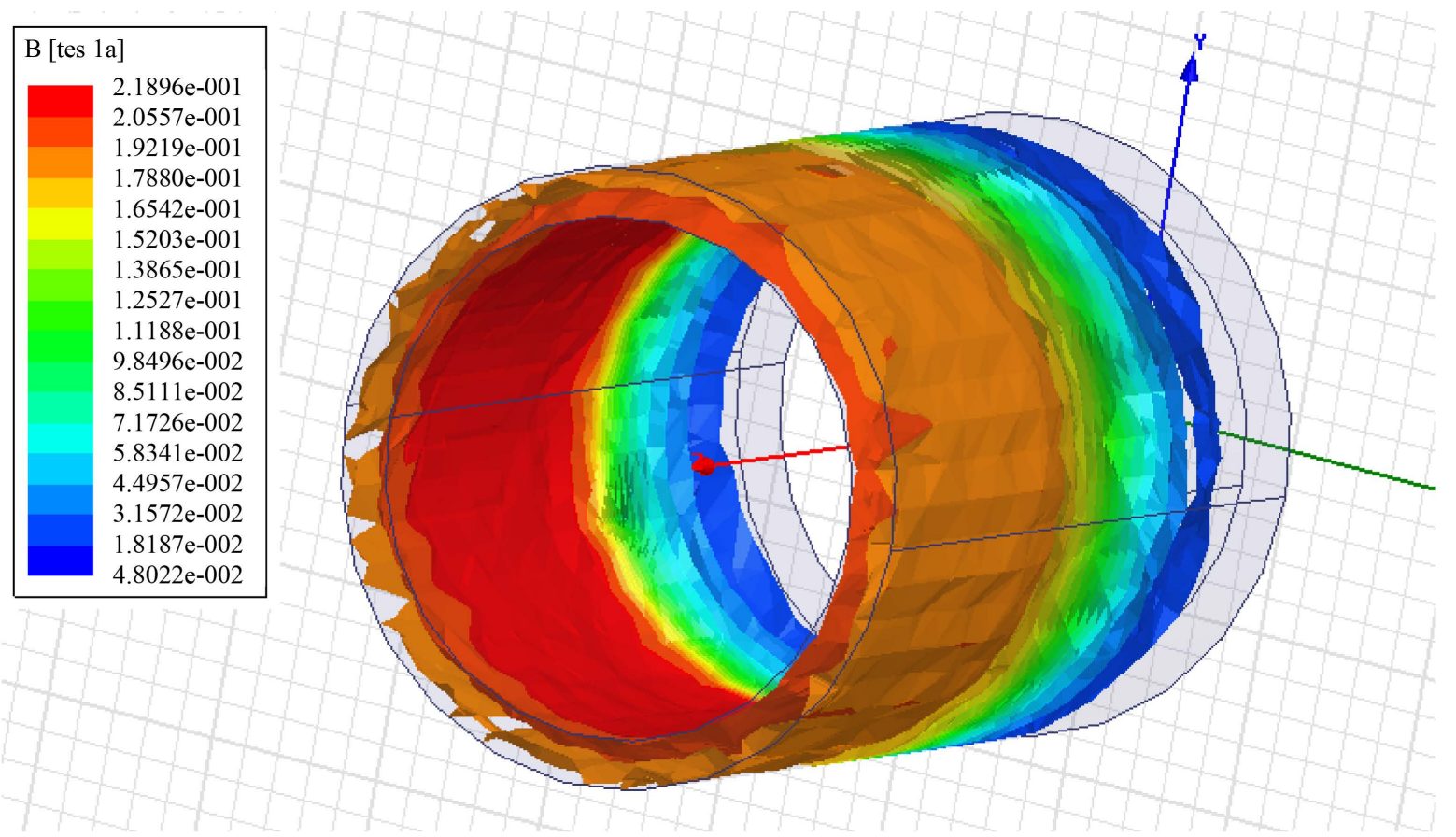

Figure 3. The simulation image of magnetic field intensity. 


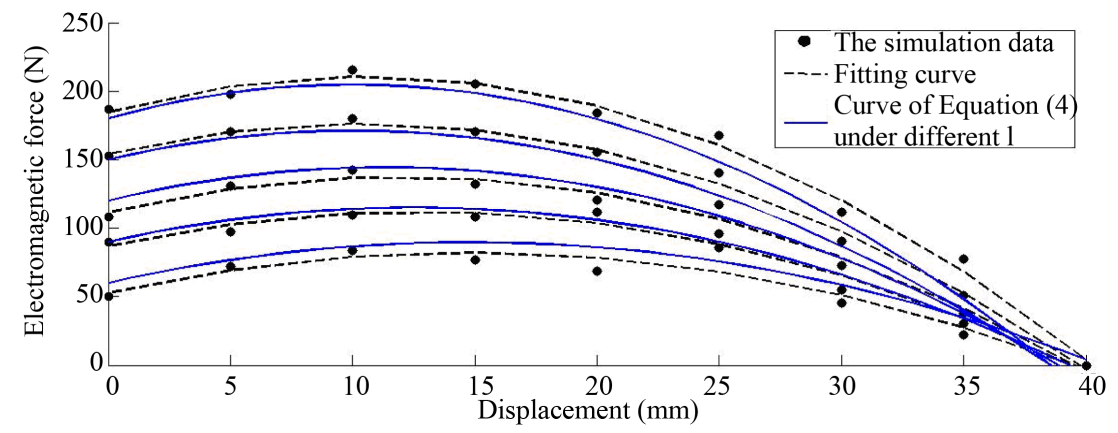

Figure 4. The simulation data, fitting curve and curve of Equation (2) under different current intensity.

\begin{tabular}{cc} 
Table 1. The main parameters of VMC. \\
\hline Parameters & Values \\
\hline Coil stroke & $40 \mathrm{~mm}$ \\
Diameter of permanent magnets & $25 \mathrm{~mm}$ \\
The resistance value & $3.7 \mathrm{ohms}$ \\
Diameter of coil & $34 \mathrm{~mm}$ \\
Width of coil & $40 \mathrm{~mm}$ \\
Inductance coefficient & $5 \mathrm{mh}$ \\
Type of permanent magnets & Nd-Fe-B (N50) \\
The maximum temperature & $428.15 \mathrm{~K}$ \\
\hline
\end{tabular}

Figure 5 shows the calculated injection pressure vs. time starting from the point of the injection $(t=0)$. The current intensity (I) of the VCM was set to 28A. The injection pressure oscillates in the first 5 ms due to the impact of the piston that causes intermittent behavior of the jet. The pressure gradually decreases in the following 10 - 100 ms. The maximum injection pressure occurs at the first peak of the oscillating curve. It is reported that the maximum injection pressure needs to reach a threshold of $14 \mathrm{MPa}$ before the jet is able to penetrate into human skin [15]. The VCM with the current intensity of 28A will produce a maximum injection pressure of 16 $\mathrm{MPa}$, which is larger than the threshold.

In order to verify the accuracy of the mathematical model, an impinging pressure measurement system was built. Due to the difficulty of fixing the mechanical structure of the system into the measurement system, the ampoule and VCM of the system were removed and were assembled into an injection device, which was then mounted onto the measurement system. Figure 6 shows the injection device.

The actual injection pressure measurement setup is shown in Figure 7. It includes a data acquisition system, an injection device that has the same system parameters with the actual system, an adjustable DC power supply $(0 \mathrm{~V}$ to $120 \mathrm{~V})(0 \mathrm{~A}$ to $36 \mathrm{~A})$ and a computer. A force transducer with frequency response of $0.1 \mathrm{~ms}$ (FSG-15N1A, Honeywell, NJ, USA) was attached to the platform. The signal is amplified and then transferred to the data collector (PCI-1711L, Advantech, Taiwan), then to the computer. The injection device has the same system parameters with the actual continuous micro-jet injection system, as shown in Table 2.

During the experiment, the output of the power supply current intensity was set to be $28 \mathrm{~A}$, and lasted for $0.01 \mathrm{~s}$. The relationships of calculated and measured injection pressure with time are put together for comparison and are shown in Figure 8. The first equation of Equation (1) follows the form of damping oscillator. It is seen from Figure 8 that while the damped frequency of the model matches the experiment, the damping ratio of the model is a bit larger. Also the peak injection pressure from calculation is about $20 \%$ larger than that obtained from the experiment. Some factors that are not included in the model such as the edge effects of the nozzle, the viscous property of the fluid, and the discrepancies of some physical properties from their actual values such as friction coefficient, may all influence the accuracy of the mathematical model.

Figure 9 shows the calculated maximum injection pressure $P_{s, \max }(\mathrm{Pa})$ increases with increasing the current intensity I(A). Its relationship can be expressed quadratically as Equation (4). 


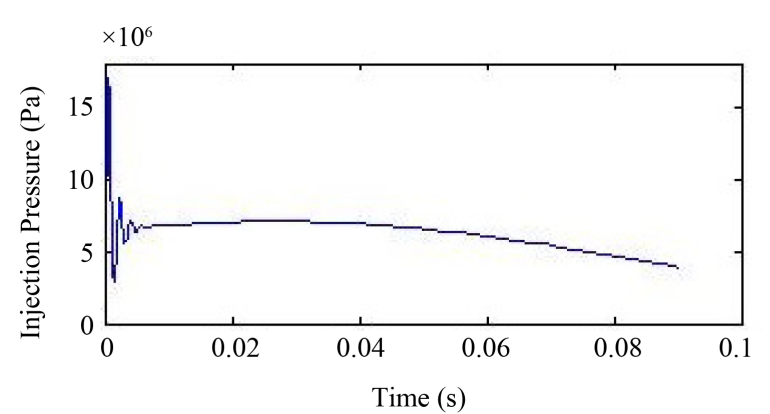

(a)

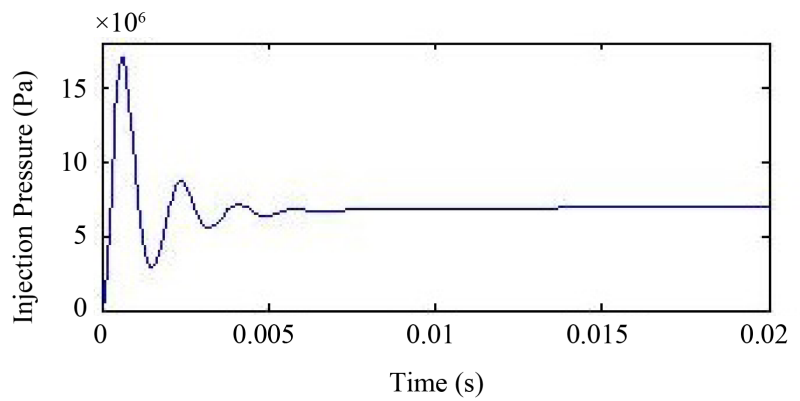

(b)

Figure 5. Calculated injection pressure vs. time (a): first $100 \mathrm{~ms}$; (b): first $20 \mathrm{~ms}$.

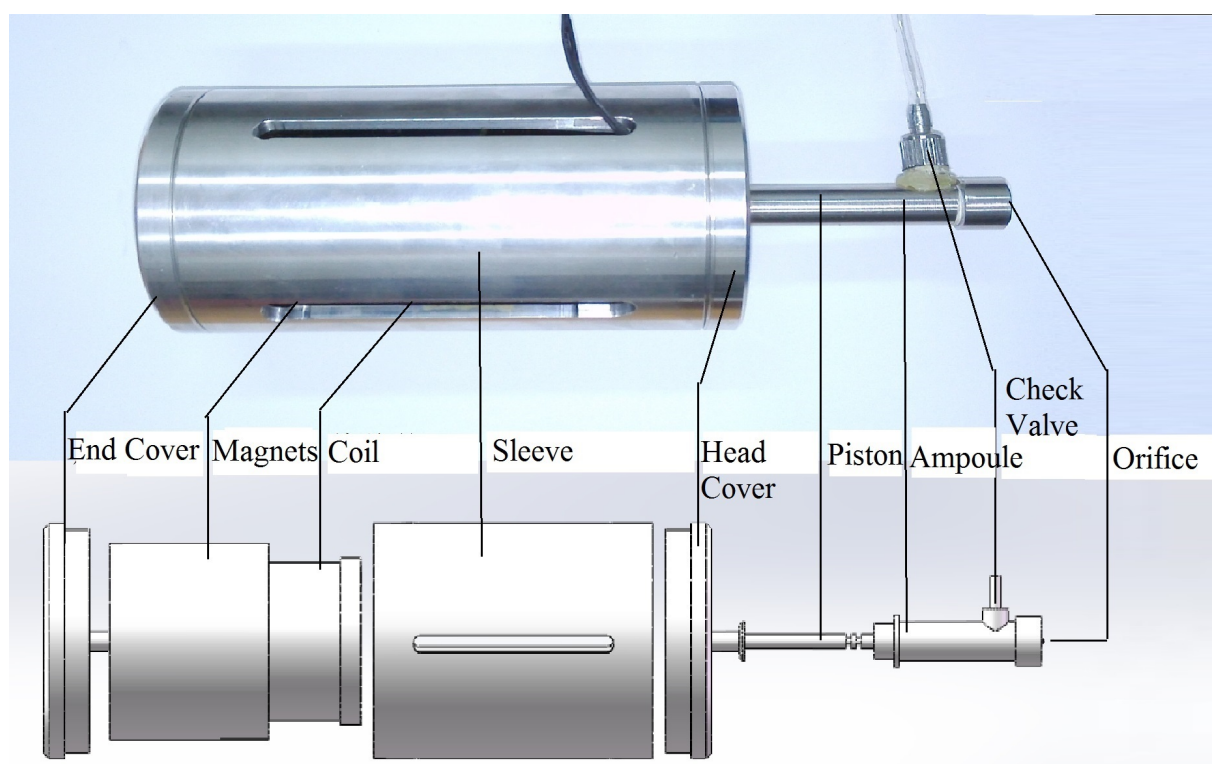

Figure 6. The injection device fitting with the pressure measurement system.

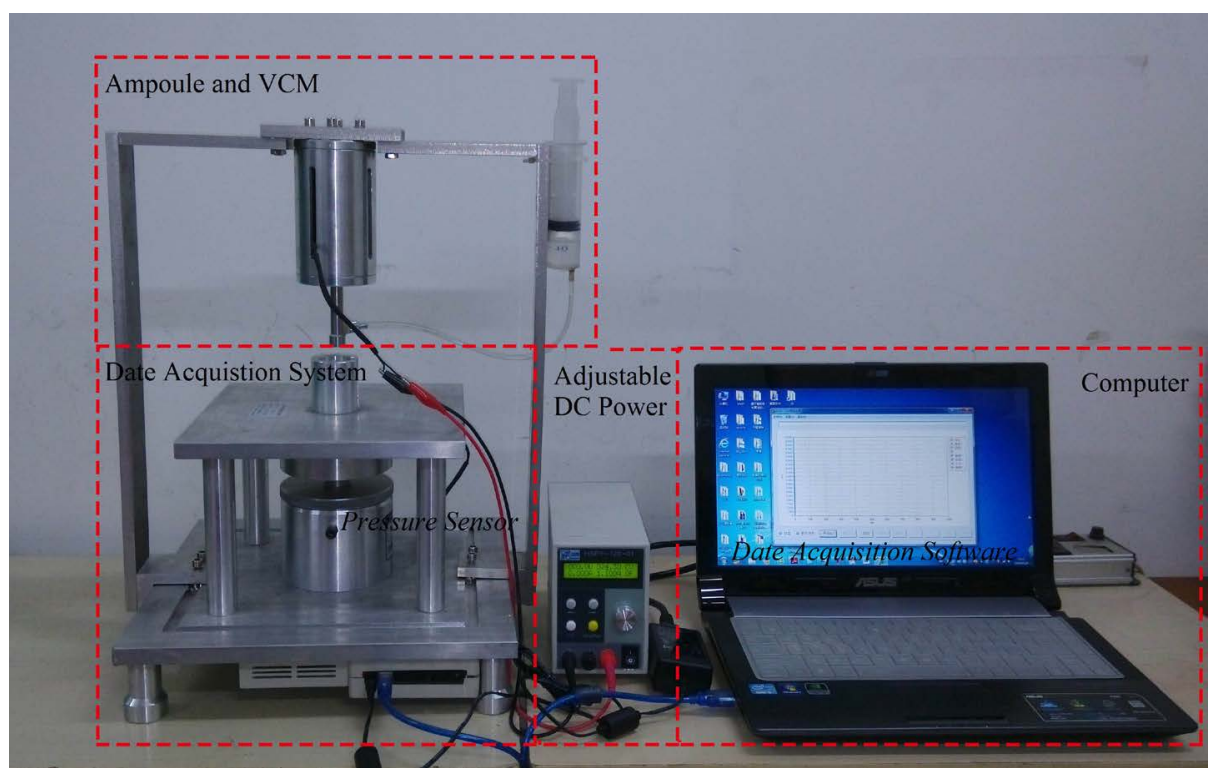

Figure 7. The actual injection pressure measurement setup. 


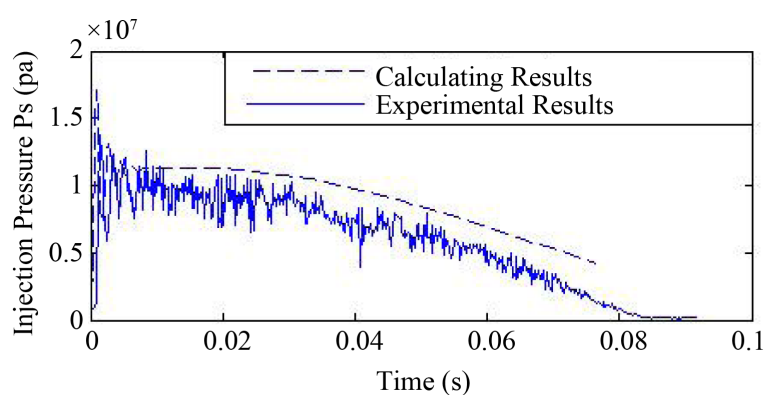

(a)

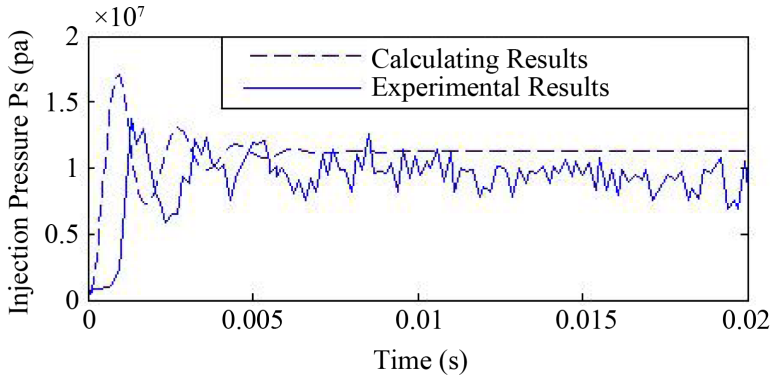

(b)

Figure 8. Injection pressure curve obtained by data acquisition software and calculated by MATLAB. (a) $0.1 \mathrm{~s}$ after the start of injection; (b) $0.02 \mathrm{~s}$ after the start of injection.

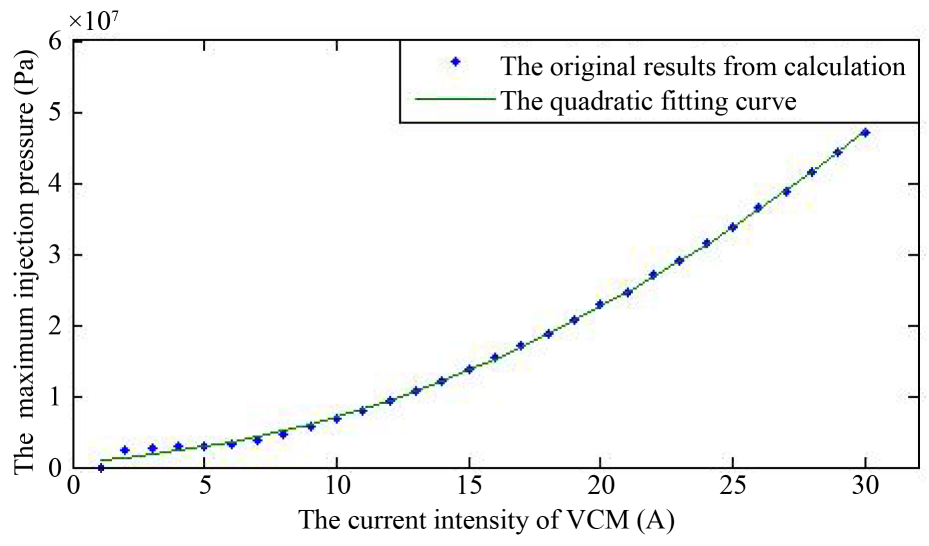

Figure 9. The maximum injection pressure vs. current intensity.

Table 2. The system parameters of the device.

Parameters
The weight of the piston and moving coil, $m_{p}$
The bulk modulus of elasticity of the drug, $E$
The initial length of liquid, $L$
The cross-sectional area of the micro orifice, $A_{o}$
The cross-sectional area of the piston, $A_{p}$
The diameter of the chamber in ampoule, $D$
The initial density of the drug, $\rho_{0}$
$4.8 \times 10^{-1} \mathrm{~kg} \times 10^{9} \mathrm{~Pa}$
$1.79 \times 10^{-8} \mathrm{~m}^{2}$
$4.77 \times 10^{-3} \mathrm{~m}$
$1 \times 10^{3} \mathrm{~kg} / \mathrm{m}^{3}$
$2 \times 10^{-2}$
The friction coefficient between ampoule and piston, $\theta$
The conventional hydraulic pressure, $p_{c}$
The contact width between the ampoule and piston, $b$
The dynamic viscosity of the drug, $\mu$

$$
P_{s, \max }=46400 I^{2}+156910 I+862040 \quad(1.05<I<35)
$$

In clinical application, each dose of pulsed jet injection can vary according to usage. The variable dose can be achieved either by fixing the ampoule chamber while changing the piston moving distance, or by fixing the piston distance while switching the ampoule. The later approach was chosen for the device and for the calculation. For each dose $(\mathrm{V})$, a try and error method was used to calculate the current intensity $(I)$ needed to keep the 
maximum injection pressure just above $16 \mathrm{Mpa}$, so that the jet has the capability of penetrating into the skin. Figure 10 shows the calculated injection pressure vs. time for a different dose of $0.2 \mathrm{ml}, 0.3 \mathrm{ml}, 0.4 \mathrm{ml}$ and 0.5 $\mathrm{ml}$ and their corresponding current intensity values so that the maximum injection pressure is above $16 \mathrm{Mpa}$.

The relation between the current intensity of VCM and the varying injection dose can be obtained by calculating each discrete value of injection dose and its corresponding value of the current intensity to maintain the maximum injection pressure right above $16 \mathrm{Mpa}$. Figure 11 shows this relationship. The current intensity first increases with the injection dose, before reaches a maximum value, and then decreases gradually. The increasing inertia is due to the increasing dose needing higher current to drive the liquid. However, the increasing cross sectional area of the ampoule due to a bigger dose will also increase the initial injection pressure, resulting in a compromising effect. The calculation results can be further expressed as a piecewise curve fitting Equation (5).

$$
\left\{\begin{array}{lc}
I=-3348.8 V^{3}+1374.1 V^{2}-104.8 V+9.9 & (0.05 \leq V \leq 0.25) \\
I=-5.3856 V+19.0666 & (0.25<V \leq 1)
\end{array}\right.
$$

Equation (4) and Equation (5) can be used for optimization of the current device to accurately control the

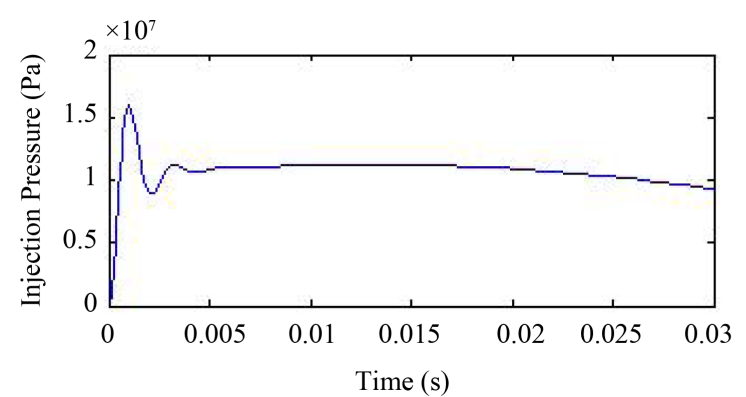

(a)

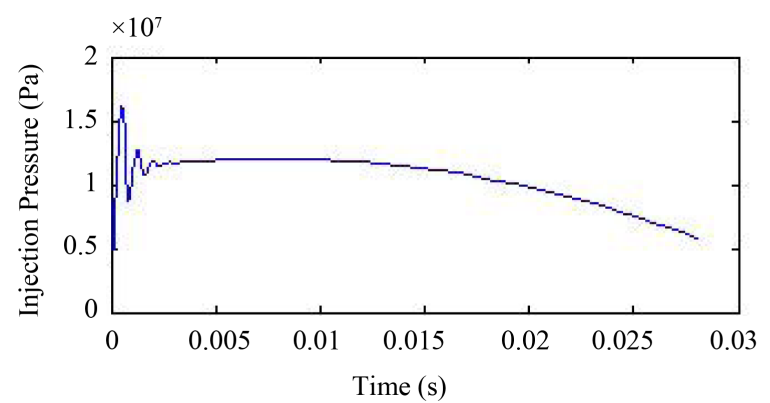

(c)

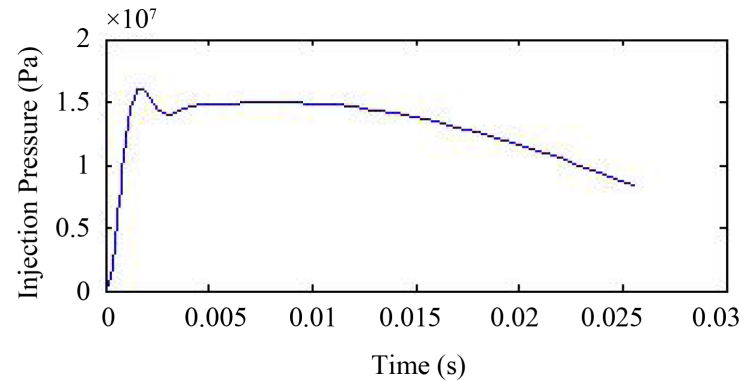

(b)

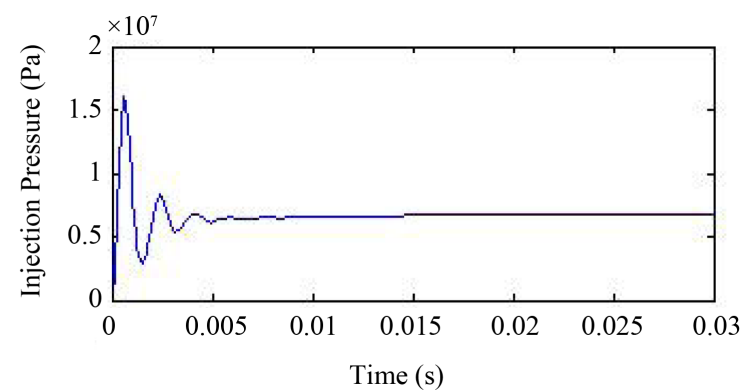

(d)

Figure 10. The injection pressure vs. time with different dose and current intensity. (a) $V=0.2 \mathrm{ml}, I=16.85^{\circ}$; (b) $V=0.3 \mathrm{ml}$, $I=17.46^{\circ}$; (c) $V=0.4 \mathrm{ml}, I=17.03^{\circ}$; (d) $V=0.5 \mathrm{ml}, I=16.37^{\circ}$.

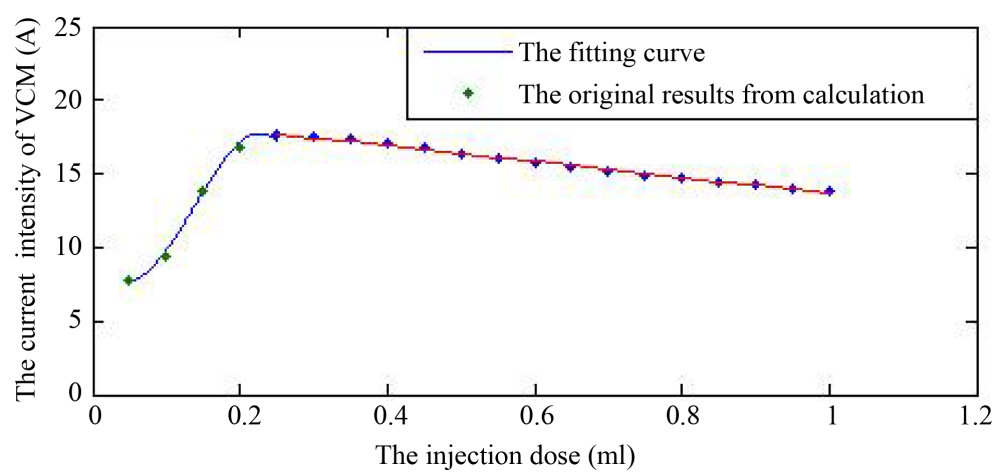

Figure 11. The relation between the injected dose and the current intensity. 
value of the current intensity of VCM for different injection doses.

Since jet injection occurs instantaneously, the process of jet-skin interaction is very difficult to be visualized. Acrylamide gel is often used to replace the human skin as an injection target for visualization of the jet injection [4] [5] [11]. Cross-sectional cut of skin after jet injection can also be viewed and used for the study of jet injection effects [12]. A different approach was used for the current study. The fluorescent imaging system was used to analyze the jet injection effects. Guinea pigs were selected for in vivo test of jet injection due to its skin property closely matching that of human skin. After skin hair removal treatment, the lower limb of an adult male Guinea pig was selected as the injection target. $0.3 \mathrm{ml}$ of Cy5 fluorescent dye (2 ug/ml) saline was injected into the subcutaneous tissue by the micro-jet injection system described in this paper. Another same kind of Guinea pig was injected with needle-syringe for the same dose of Cy5 fluorescent dye as control group. The Guinea pig was placed in a Multi-Spectral In-Vivo Imaging System for analysis (Maestro 1 from Caliper Life Sciences, MA, USA, with $650 \mathrm{~nm}$ excitation wavelength) immediately after being injected. The fluorescence distribution around the injection point was observed and sampling images were taken for discrete time periods. It was found that fluorescence concentration focused on the injection point for a long period (10 minutes) after injection with needle syringe, whereas fluorescence dye using the current micro-jet injection system has spread to the lower extremity, lower extremity, partial abdominal injections, as well as both upper limbs even after 1 minute of injection, and spread to almost everywhere after 30 minutes (show in Figure 12). The results show that, the current micro-jet injection system based on a voice coil linear driver has been able to perform subcutaneous injection in Guinea pig, and the biological absorption rate through the jet injection system for subcutaneous injection is much faster than that using hypodermic needle. Further experiments will be carried out in the future to verify the calculation results of varying dose situation and the effects of pulsed injection of the device.

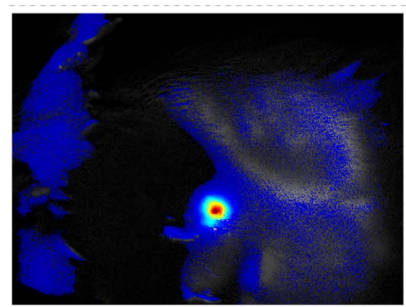

$1 \mathrm{~min}$

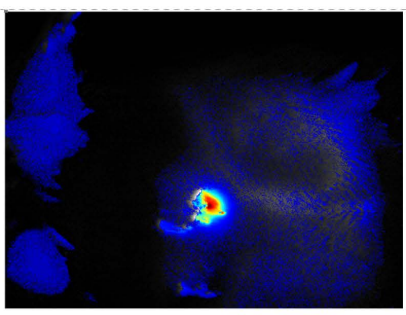

$10 \mathrm{~min}$

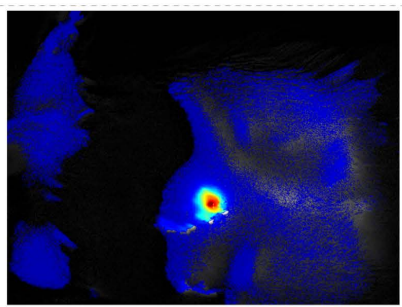

$5 \mathrm{~min}$

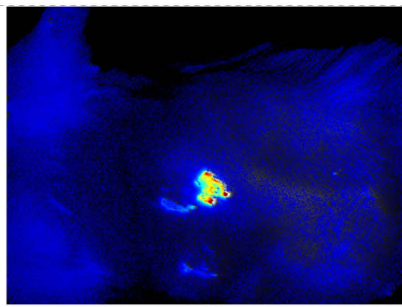

$30 \mathrm{~min}$

(a)

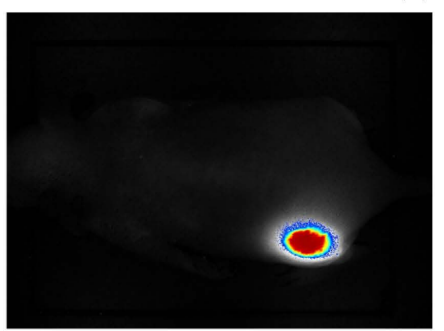

$1 \mathrm{~min}$

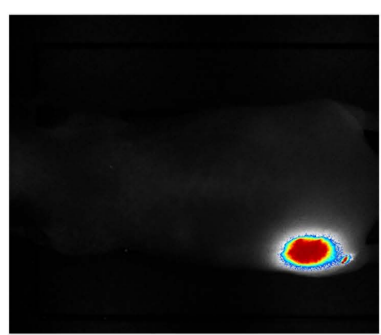

$10 \min$

(b)

Figure 12. Fluorescent images of injection by micro-jet injection (a) and hypodermic needle (b) in Guinea pigs. 


\section{Conclusions}

A controllable micro-jet injection system powered by a Lorentz force driver (or voice coil motor, VCM) was presented in this paper. The device is able to perform a continuous pulsed jet injection through the control of electrical current passing through the VCM. The analysis of the jet mechanics was carried out based on the existing physical model in order to establish the relationship between the controllable current intensity and the injection pressure under different system parameters. The following conclusions can be obtained:

1) The driving force (Lorentz force of VCM) was simulated by FEM and can be represented through a function of current intensity under fixed system conditions. It can be substituted into the existing physical model to obtain the injection pressure under different system conditions. The calculated injection pressure matches the experimental measurement under same system conditions.

2) An in vivo injection test was performed on Guinea pig using the current micro-jet injection system and a needle syringe. The results show the current system is able to inject into Guinea pig subcutaneously and the absorption rate of solution is much faster than injection by hypodermic needle.

3) The relationship between the maximum injection pressure that characterizes the jet performance and the current intensity was established, and can be represented through a curve fitting function.

4) In a variable-dose situation, the relationship between the current intensity and the value of dose was obtained from calculation to keep the maximum injection pressure above a threshold. The above relationships can be used for optimization of the micro-jet injection device.

\section{Acknowledgements}

The current project is under support of China's National Science Foundation (No. 51275142 and No. 31271002).

\section{References}

[1] Prausnitz, M.R., Mitragotri, S. and Langer, R. (2004) Current Status and Future Potential of Transdermal Drug Delivery. Nature Reviews Drug Discovery, 3, 115-124. http://dx.doi.org/10.1038/nrd1304

[2] Arora, A., Hakim, I., Baxter, J., Rathnasingham, R., Srinivasan, R., Fletcher, D.A. and Mitragotri, S. (2007) NeedleFree Delivery of Macromolecules across the Skin by Nanoliter-Volume Pulsed Microjets. Proceedings of the National Academy of Sciences of the United States of America, 14, 4255-4260. http://dx.doi.org/10.1073/pnas.0700182104

[3] Arora, A., Prausnitz, M.R. and Mitragotri, S. (2008) Micro-Scale Devices for Transdermal Drug Delivery. International Journal of Pharmaceutics, 364, 227-236. http://dx.doi.org/10.1016/j.ijpharm.2008.08.032

[4] Stachowiak, J.C., von Muhlen, M.G., Li, T.H. and Fletcher, D.A. (2007) Piezoelectric Control of Needle-Free Transdermal Drug Delivery. Journal of Controlled Release, 124, 88-97. http://dx.doi.org/10.1016/j.jconrel.2007.08.017

[5] Stachowiak, J.C., Li, T.H., Arora, A., Mitragotri, S. and Fletcher, D.A. (2009) Dynamic Control of Needle-Free Jet Injection. Journal of Controlled Release, 135, 104-112. http://dx.doi.org/10.1016/j.jconrel.2009.01.003

[6] Chen, K. (2009) A Novel Micro-Jet Transdermal Drug Delivery System. Proceedings of ASME 4th Frontiers of Biomedical Devices, Irvine, CA, 8-9 June 2009, 67-68.

[7] Fletcher, D.A. and Palanker, D.V. (2001) A Pulsed Liquid Microjet for Ocular Microsurgical Applications. Journal of Investigative Ophthalmology \& Visual Science, 42, S717.

[8] Fletcher, D.A., Palanker, D.V., Huie, P., Miller, J., Marmor, M. and Brumenkranz, M.S. (2002) Intravascular Drug Delivery with a Pulsed Liquid Microjet. Archives of Ophthalmology, 120, 1206-1208. http://dx.doi.org/10.1001/archopht.120.9.1206

[9] Han, T.H. and Yoh, J.J. (2010) A Laser Based Reusable Microjet Injector for Transdermal Drug Delivery. Journal of Applied Physics, 107, 103110-103113. http://dx.doi.org/10.1063/1.3430989

[10] Taberner, A.J., Ball, N.B., Hogan, N.C. and Hunter, I.W. (2006) A Portable Needle-Free Jet Injector Based on a Custom High Power-Density Voice-Coil Actuator. 28th Annual International Conference of the IEEE Engineering in Medicine and Biology Society, New York, 30 August-3 September 2006, 5001-5004. http://dx.doi.org/10.1109/iembs.2006.260243

[11] Taberner, A.J., Hogan, N.C. and Hunter, I.W. (2012) Needle-Free Jet Injection Using Real-Time Controlled Linear Lorentz-Force Actuators. Medical Engineering \& Physics, 34, 1228-1235. http://dx.doi.org/10.1016/j.medengphy.2011.12.010

[12] Schramm-Baxter, J. and Mitragotri, S. (2004) Needle-Free Jet Injections: Dependence of Jet Penetration and Disper- 
sion in the Skin on Jet Power. Journal of Controlled Release, 97, 527-535.

http://dx.doi.org/10.1016/j.jconrel.2004.04.006

[13] Baxter, J. and Mitragotri, S. (2005) Jet-Induced Skin Puncture and Its Impact on Needle-Free Jet Injections: Experimental Studies and a Predictive Model. Journal of Controlled Release, 106, 361-373.

http://dx.doi.org/10.1016/j.jconrel.2005.05.023

[14] Chen, K., Zhou, H., Li, J. and Cheng, G.J. (2010) A Model on Liquid Penetration into Soft Material with Application to Needle-Free Jet Injection. ASME Journal of Biomechanical Engineering, 132, 101005-1.

[15] Shergold, O.A., Fleck, N.A. and King, T.S. (2005) The Penetration of a Soft Solid by a Liquid Jet, with Application to the Administration of a Needle-Free Injection. Journal of Biomechanics, 39, 2593-2602. http://dx.doi.org/10.1016/i.jbiomech.2005.08.028

[16] Chen, K., Zhou, H., Li, J. and Cheng, G. (2011) Stagnation Pressure in Liquid Needle-Free Injection: Modeling and Experimental Validation. Drug Delivery Letters, 1, 97-104. http://dx.doi.org/10.2174/2210303111101020097

[17] Xu, Q.W., Chen, K. and Lv, Y.G. (2015) FEM Modeling and Validation of a Voice Coil Powered Jet Injector. Proceedings of Asia-Pacific Biological Engineering and Biomedical Conference, Shanghai, 30-31 January 2015, $207-212$. 